QUARTERLY OF APPLIED MATHEMATICS

VOLUME LXVIII, NUMBER 1

MARCH 2010, PAGES 81-90

S 0033-569X(09)01166-1

Article electronically published on October 15, 2009

\title{
ON THE PARTICLE PATHS IN SOLITARY WATER WAVES
}

\author{
BY \\ ADRIAN CONSTANTIN \\ School of Mathematics, Trinity College, Dublin 2, Ireland \\ Dedicated to Walter Strauss on his 70th birthday with esteem and friendship.
}

\begin{abstract}
We provide the qualitative flow pattern beneath a solitary water wave by describing the individual particle trajectories.

Preamble. Hydrodynamics is a research field where Walter Strauss is recognized throughout the world for some groundbreaking contributions. It is also an area of Strauss's work I have had the privilege and pleasure to participate in. The present paper is devoted to one of the most striking manifestations of water waves: solitary waves. Adapting to the context of solitary waves some considerations made for periodic traveling waves in a recent joint work with Strauss [19, we will provide the qualitative flow pattern beneath a solitary water wave by describing the individual particle trajectories, offering a different and simpler approach to the recent results in [11.
\end{abstract}

1. Introduction. Solitary water waves are localized nonlinear waves that propagate steadily. They were first noticed by Scott Russell in 1834, as described in a now famous report on his observations of a large heap of water traveling with undiminished speed or shape over a distance along a Scottish canal [38:

"I believe I shall best introduce the phaenomenon by describing the circumstances of my own first acquaintance with it. I was observing the motion of a boat which was rapidly drawn along a narrow channel by a pair of horses, when the boat suddenly stopped - not so the mass of water in the channel which it had put in motion; it accumulated round the prow of the vessel in a state of violent agitation, then suddenly leaving it behind, rolled forward with great velocity, assuming the form of a large solitary elevation, a rounded, smooth and well-defined heap of

Received December 12, 2008.

2000 Mathematics Subject Classification. Primary 35Q35, 76B07; Secondary 35J65, 76B25.

Key words and phrases. Euler equations, free boundary, conformal map, particle trajectory.

Current address: University of Vienna, Fakultät für Mathematik, Nordbergstraße 15, 1090 Wien, Austria.

E-mail address: adrian.constantin@univie.ac.at 
water, which continued its course along the channel apparently without change of form or diminution of speed. I followed it on horseback, and overtook it still rolling on at a rate of some eight or nine miles an hour, preserving its original figure some thirty feet long and a foot to a foot and a half in height. Such, in the month of August, 1834, was my first chance interview with that singular and beautiful phaenomenon."

The ability of these water waves to retain their shape for a long period of time is quite remarkable. Subsequent to his observation, Russell conducted numerous detailed experiments [38] to investigate the nature of what he called "the great wave of translation", but which came to be known as the solitary wave. By measuring the velocity and height of the solitary waves, Russell established phenomenological relations connecting these quantities which have stood the test of time (see 31]). Some forty years later theoretical work by Boussinesq [6] and Rayleigh [37] established an analytical model for irrotational gravity waves of small amplitude in the shallow water/long-wave regime. Then in 1895 Korteweg and de Vries [33] derived the well-known equation which now bears their names as a model for irrotational solitary shallow water waves of small amplitude. Significant further developments had to wait until the second half of the twentieth century, when the investigation of solitary water waves of large amplitude was initiated. The linear theory of water waves of small amplitude fails to yield any reasonable approximation to solitary waves 39 and for this reason even the earliest models for solitary waves had to incorporate nonlinear effect:1 1 . The simplest effective nonlinear approximation to the governing equations for water waves in the shallow water limit is the Korteweg-de Vries $(\mathrm{KdV})$ equation [33: for small-amplitude long waves there always exists a region in space-time where a balance between nonlinearity and dispersion generates as a leading order approximation to the governing equations for water waves the KdV equation (see the discussion in [12). The solitary wave solutions of KdV have remarkable features: they are solitons, recovering their shape and speed through nonlinear interactions, a fact indicating the rich structure of $\mathrm{KdV}$ as an integrable infinite-dimensional Hamiltonian system [23. While much of the current interest in water waves was stirred by the success of soliton theory [31, one should emphasize that KdV and other subsequently derived integrable or non-integrable model equations for shallow water waves are valid approximations only for waves of small amplitude (see e.g. the discussion in [14). To understand features of solitary waves of large amplitude one cannot rely on such model equations and a direct study of the governing equations for water waves appears to be unavoidable. A rigorous study of solitary wave solutions to the governing equations for irrotational water waves was initiated by Friedrichs and Hyers [26] and subsequently improved by Beale [5], with further substantial contributions due to Amick, Craig, and

\footnotetext{
${ }^{1}$ In the early days, the existence of solitary waves excited some controversy since Russell's conclusions contradicted some predictions of Airy [1], who was considered at that moment to be the leading expert in hydrodynamics; see 22 for interesting historical information about the origins of water wave theory. The insight of Boussinesq 6] and, independently, of Rayleigh [37, was to incorporate weak nonlinear effects, in particular an appropriate allowance for vertical acceleration which had been neglected in the linear theory developed by Airy (see the discussion in [35]).
} 
Toland [2, 3, 4, 20, 21, 40]. Despite all these results, there are no explicit formulas available for these wave patterns - a situation that unfortunately seems to be characteristic of the governing equations, the few notable exceptions all being rather special flows with peculiar vorticities [7, 8, 27, 29, 36. Therefore further progress depends on qualitative studies of the governing equations, rather than on deriving and investigating model equations for small perturbations of certain flows, in contrast to the setting in which KdV rose to prominence, which is appropriate for small perturbations of a flat water surface. One of the most fundamental questions related to solitary water waves is the elucidation of the flow pattern beneath the wave. Recently, there has been progress in this direction [11, and it is our aim to present here a slightly different viewpoint from that advocated in [11] in order to obtain a setting which allows us to simplify the proofs of the main results in [11].

2. Preliminaries. We now describe the governing equations for gravity water waves and recall some basic information available on their solitary wave solutions.

2.1. The governing equations for irrotational two-dimensional gravity water waves. Consider the one-dimensional propagation (in the $x$-direction) of a wave with uniform cross-section, at the surface of a flat-bed layer of an incompressible and inviscid fluid (water). Let $y=0$ be the flat bed and let us denote by $y=\eta(x, t)>0$ the water's free surface. The fluid motion can be represented by the velocity vector $(u(x, y, t), v(x, y, t))$, where $u$ and $v$ are the horizontal and vertical velocity components, respectively.

It is physically realistic to take the density of water to be constant 34 so that incompressibility is expressed by the equation of mass conservation

$$
u_{x}+v_{y}=0 .
$$

The equation of motion is Euler's equation

$$
\left\{\begin{array}{l}
u_{t}+u u_{x}+v u_{y}=-P_{x} \\
v_{t}+u v_{x}+v v_{y}=-P_{y}-g
\end{array}\right.
$$

where $P(t, x, y)$ is the hydrodynamical pressure in the fluid and $g$ is the (constant) acceleration of gravity. Euler's equation (2.2) encompasses a number of reasonable assumptions for the study of water waves of large amplitude [34]:

- once the water motion has been set up, the only external force is gravity, acting vertically downwards, so that the balance between the restoring gravity force and the inertia of the system governs the evolution of the disturbance of the water surface;

- viscosity is neglected so that the effect of the internal forces is entirely encoded in the hydrodynamical pressure $P(t, x, y)$.

The vorticity of a flow, $\omega=v_{x}-u_{y}$, measures the local whirl of a fluid element. An important category of flows comprises the irrotational flows, characterized by the additional assumption

$$
u_{y}=v_{x}
$$

of zero vorticity. Concerning the physical relevance of irrotational water flows, experimental evidence indicates that for sea waves entering a region of still water the assumption of 
irrotational flow is realistic [34. Moreover, a water flow that is irrotational initially has to be irrotational at all later times [31. For this reason, in the absence of a underlying current the irrotationality of the flow is usually assumed, as we shall do throughout this paper.

The above equations (2.1), (2.2) and (2.3) have to be supplemented by appropriate boundary conditions, taking into account the fact that the horizontal bed at $y=0$ is rigid and that the upper boundary of the fluid, $y=\eta(x, t)$, is a free surface. As a result, the free surface decouples the motion of the water from that of the air above so that the dynamic boundary condition

$$
P=P_{0} \quad \text { on } \quad y=\eta(x, t)
$$

must hold if we neglect surface tension, where $P_{0}$ is the (constant) atmospheric pressure. Moreover, since the same particles always form the free surface, we also have the kinematic boundary condition

$$
v=\eta_{t}+u \eta_{x} \quad \text { on } \quad y=\eta(x, t) .
$$

On the flat bed we have the kinematic boundary condition

$$
v=0 \quad \text { on } \quad y=0
$$

expressing the fact that water cannot penetrate the rigid bed. The governing equations for irrotational two-dimensional gravity water waves are (2.1)-(2.6). All of the functions involved are smooth.

2.2. Solitary irrotational water waves. Among the various solutions to the governing equations for irrotational two-dimensional gravity water waves, the solitary waves are characterized by being localized traveling waves. That is, given the constant wave speed $c>0$, solitary waves traveling at speed $c$ are found by requiring that all smooth functions $u, v, P, \eta$ exhibit an $(x, t)$-dependence in the form of $(x-c t)$ and that, in addition, as $x \rightarrow \pm \infty$ the flow is at rest and the free surface approaches a height $h>0$ above the flat bed. The existence of solitary waves of small amplitude was first proved by a power series method in [26, with a later approach using the implicit function theorem [5], while the existence of solitary waves of large amplitude (up to the wave of greatest height) was obtained using global bifurcation theory [3, 4,. One can show 2 that the inequality

$$
c>\sqrt{g h}
$$

must hold for all genuine solitary waves (that is, without a flat surface) and that all these waves are symmetric waves of elevation with a single wave crest [21]:

- the waves are symmetric waves of elevation, that is,

$$
\begin{gathered}
\eta(x)>h \text { and } \eta\left(x_{0}-x\right)=\eta\left(x_{0}+x\right) \quad \text { for all } \quad x \in \mathbb{R}, \\
u\left(x_{0}-x, y\right)=u\left(x_{0}+x, y\right) \quad \text { for all } x \in \mathbb{R}, \quad 0 \leq y \leq \eta(x), \\
v\left(x_{0}-x, y\right)=-v\left(x_{0}+x, y\right) \quad \text { for all } \quad x \in \mathbb{R}, \quad 0 \leq y \leq \eta(x),
\end{gathered}
$$

where $x_{0}$ is the location of the wave crest;

- the wave profile $x \mapsto \eta(x)$ is strictly monotone on either side of the wave crest — in particular, there is a single wave crest. 
All solitary waves are smooth, except the wave of greatest height [3, 4], and we restrict our attention to the smooth solitary waves; for the special case of the solitary waves of greatest height our approach is not applicable, so we refer to [11 for a description of the flow pattern beneath such waves. For all smooth solitary waves the inequality

$$
u<c
$$

holds inside the fluid as well as on the upper and lower boundaries of the fluid domain [40, and establishes the experimentally verifiable fact 34] that the wave speed exceeds the horizontal velocity of any water particle. The inequality (2.8) is fundamental to our considerations and this is the reason why the approach pursued here does not extend to the case of the solitary waves of greatest height, since for these waves $u=c$ at the wave crest (see the discussion in [11]).

It is convenient to reformulate the governing equations for irrotational solitary waves in terms of the velocity potential and the stream function. Taking advantage of (2.3), we define the velocity potential $\phi$ by

$$
\phi_{x}=u-c, \quad \phi_{y}=v, \quad \phi(0,0)=0,
$$

that is,

$$
\phi(x, y)=\int_{0}^{x}[u(l, 0)-c] d l+\int_{0}^{y} v(x, s) d s .
$$

On the other hand, using (2.1), we define the stream function $\psi$ by

$$
\psi_{x}=-v, \quad \psi_{y}=u-c, \quad \psi(0, \eta(0))=0,
$$

that is,

$$
\psi(x, y)=m-\int_{0}^{x} v(l, 0) d l+\int_{0}^{y}[u(x, s)-c] d s
$$

for a suitable constant $m \in \mathbb{R}$. In view of (2.10) and (2.5)-(2.6), we see that $\psi$ is constant on the flat bed $y=0$ as well as on the free surface:

$$
\psi(x, 0)=m, \quad \psi(x, \eta(x))=0, \quad x \in \mathbb{R} .
$$

Note the relation

$$
m=-\int_{0}^{\eta(x)} \psi_{y}(x, y) d y=\int_{0}^{\eta(x)}[c-u(x, y)] d y, \quad x \in \mathbb{R},
$$

which substantiates the definition of $m$ as the relative mass flux of the flow associated with the solitary wave, and ensures that $m>0$ in view of (2.8).

The functions $\phi$ and $\psi$ are harmonic conjugated, in the sense that the complex function

$$
(x+i y) \mapsto \phi(x, y)+i \psi(x, y)
$$

is analytic on its domain of definition. This fundamental property is a consequence of (2.1), (2.3) and (2.9), (2.10). A further important observation is that one can recast the Euler equation (2.2) as stating that the expression

$$
\frac{(u-c)^{2}+v^{2}}{2}+P+g y
$$


is constant throughout the fluid. The asymptotic form of the flow as $x \rightarrow \pm \infty$ allows us to find the value of this constant by evaluating the expression on the free surface $y=\eta(x)$ in the limit $x \rightarrow \infty$, taking into account also the boundary condition (2.4). We find that

$$
\frac{(u-c)^{2}+v^{2}}{2}+P+g y=\frac{c^{2}}{2}+P_{0}+g h, \quad x \in \mathbb{R}, \quad 0 \leq y \leq \eta(x) .
$$

These considerations show that the governing equations for irrotational solitary waves lead us to look for a harmonic function $\psi$ satisfying the nonlinear time-independent free boundary problem

$$
\left\{\begin{array}{l}
\Delta \psi=0, \quad x \in \mathbb{R}, \quad 0 \leq y \leq \eta(x), \\
|\nabla \psi(x, \eta(x))|^{2}+2 g[\eta(x)-h]=c^{2}, \quad x \in \mathbb{R}, \\
\psi(x, \eta(x))=0, \quad x \in \mathbb{R}, \\
\psi(x, 0)=m, \quad x \in \mathbb{R},
\end{array}\right.
$$

coupled with the asymptotic limits

$$
\left\{\begin{array}{l}
\lim _{|x| \rightarrow \infty} \eta(x)=h, \\
\lim _{|x| \rightarrow \infty} \nabla \psi(x, y)=(-c, 0) \quad \text { uniformly for } 0 \leq y \leq \eta(x) .
\end{array}\right.
$$

An interesting and useful fact is that along the free surface $y=\eta(x)$ the horizontal velocity $u$ is strictly monotone on either side of the crest, as a function of $x$, with the maximum attained at the crest. To show this, notice first that using (2.2) and (2.10) we can compute

$$
\Delta P=-2 \psi_{x y}^{2}-2 \psi_{x x}^{2} \leq 0 .
$$

The second equation in (2.2) and (2.6) yield $P_{y}(x, 0)=-g$ for all $x \in \mathbb{R}$. On the other hand, the fact that asymptotically the flow is at rest ensures by (2.13) that $P(x, y)$ approaches the hydrostatic pressure $P_{0}+g(h-y)$ for $|x| \rightarrow \infty$, exceeding therefore the value $P_{0}$ beneath the free surface in view of the first relation in (2.15). Therefore Hopf's maximum principle [25] for the superharmonic function $P$ yields that the minimum of $P$ is attained all along the free surface $y=\eta(x)$, where $P=P_{0}$ by (2.4). A new application of Hopf's maximum principle yields now that $P_{x}(x, \eta(x))>0$ for $x<x_{0}$ while $P_{x}(x, \eta(x))<0$ for $x>x_{0}$, since the surface profile rises monotonically towards the crest $\left(x_{0}, \eta\left(x_{0}\right)\right)$. Since $P_{x}=(c-u)\left[u_{x}+\eta_{x} u_{y}\right]$ on $y=\eta(x)$ by the first equation in (2.2) as $v=(u-c) \eta_{x}$ along the free surface in view of (2.5), we deduce that

$$
\left\{\begin{array}{llll}
\frac{d}{d x} u(x, \eta(x))=u_{x}-\frac{v}{c-u} u_{y}>0 & \text { along } \quad y=\eta(x) \text { for } \quad x<x_{0}, \\
\frac{d}{d x} u(x, \eta(x))=u_{x}-\frac{v}{c-u} u_{y}<0 & \text { along } y=\eta(x) \text { for } \quad x>x_{0} .
\end{array}\right.
$$

3. Main result. We now describe the flow pattern beneath a smooth solitary wave.

THEOREM 3.1. If a nontrivial smooth solitary wave propagates in irrotational flow at the free surface of water with a flat bed, then the water particles on the flat bed move horizontally with positive speed in the direction of wave propagation. A water particle above the flat bed, whether beneath or on the free surface, moves always in the direction 
of wave propagation in such a way that the wave crest is located straight above it (or at it) at precisely one instant of time. Prior to this instant the particle moves up, and afterwards it moves down.

Proof. We first prove that at any instant, beneath a nontrivial smooth solitary wave, i.e. one with a non-flat water surface, we must have that $u>0$ throughout the fluid while $v=0$ just on the flat bed and straight below the wave crest, and elsewhere $v>0$ in front of the vertical segment below the wave crest and $v<0$ behind it.

To see this, assume for simplicity that the wave crest is located at $(0, \eta(0))$. The conformal hodograph transform

$$
\left\{\begin{array}{l}
q=-\phi(x, y) \\
p=-\psi(x, y)
\end{array}\right.
$$

transforms the free boundary problem (2.14) into the nonlinear boundary problem

$$
\left\{\begin{array}{lr}
\Delta_{q, p} H=0, & q \in \mathbb{R}, \quad-m \leq p \leq 0, \\
H(q,-m)=-h, & q \in \mathbb{R}, \\
\left(H_{q}^{2}(q, 0)+H_{p}^{2}(q, 0)\right)\left(c^{2}-2 g H(q, 0)\right)=1, & q \in \mathbb{R},
\end{array}\right.
$$

with the asymptotic limit

$$
\left\{\begin{array}{l}
\lim _{|q| \rightarrow \infty} H(q, 0)=0, \\
\lim _{|q| \rightarrow \infty} \nabla_{q, p} H(q, p)=\left(0, \frac{1}{c}\right) \quad \text { uniformly for } \quad-m \leq p \leq 0,
\end{array}\right.
$$

for the harmonic function

$$
H(q, p)=y-h .
$$

It is useful to notice that

$$
H_{q}=-\frac{v}{(c-u)^{2}+v^{2}}, \quad H_{p}=\frac{c-u}{(c-u)^{2}+v^{2}} .
$$

Since $v=(u-c) \eta_{x}$ along $y=\eta(x)$ by (2.5), the monotonicity properties of the free surface in combination with (2.8) and (3.5) yield

$$
H_{q}(q, 0)>0 \quad \text { for } \quad q<0, \quad H_{q}(q, 0)<0 \quad \text { for } \quad q>0 .
$$

On the other hand, the symmetry of the flow yields $v(0, y)=0$ for $0 \leq y \leq \eta(0)$, so that (3.5) yields

$$
H_{q}(0, p)=0, \quad-m \leq p \leq 0,
$$

while $H_{q}(q,-m)=0$ in view of the second equation in (3.2). The first equation in (3.3) and the strong maximum principle [25. for the harmonic function $H$ together with (3.5) lead us to the claimed property of the vertical velocity $v$.

Since the hodograph transform is conformal, the harmonic function $u(x, y)$ remains harmonic if viewed as a function of $(q, p)$. Thus $u_{q}$ is harmonic in the half-strip $\{(q, p)$ : $q \leq 0,-m \leq p \leq 0\}$. As

$$
\partial_{q}=H_{p} \partial_{x}+H_{q} \partial_{y}
$$


for the performed change of variables, (3.5) yields

$$
u_{q}=\frac{(c-u) u_{x}-v u_{y}}{(c-u)^{2}+v^{2}} .
$$

Now the half-line $\{(q,-m): q<0\}$ corresponds to the half-line $\{(x, 0): x<0\}$, so $u_{q}(q,-m)>0$ for $q<0$. Indeed,

$$
u_{q}(q,-m)=-\frac{v_{y}(x, 0)}{c-u(x, 0)}
$$

in view of (2.6) and (2.1), and on the one hand we have (2.8) while on the other hand $v_{y}(x, 0)<0$ by Hopf's maximum principle [25] since the harmonic function $v$ attains in the region $\{(x, y): x \leq 0,0 \leq y \leq \eta(x)\}$ its maximum on $y=0$. On the vertical segment $q=0$ we have $u_{q}=0$, as $v_{p}=0$ there (since $v=0$ there) and $(u, v)$ are harmonic conjugate functions. Combining this information with (2.16) and (3.6), we conclude by the maximum principle that $u_{q}>0$ for $q<0$, since asymptotically $u, u_{q} \rightarrow 0$ holds. Similarly $u_{q}<0$ for $q>0$. In particular, since $u \rightarrow 0$ asymptotically, we deduce that $u>0$ throughout the flow.

Since the particle paths $(x(t), y(t))$ are obtained as the solutions to the system

$$
\left\{\begin{array}{l}
x^{\prime}=u(x-c t, y) \\
y^{\prime}=v(x-c t, y)
\end{array}\right.
$$

the above considerations interpreted in terms of $x(t)$, respectively $y(t)$, complete the proof.

REMARK 3.2. As already pointed out in Section 2, our approach is not directly applicable to the solitary waves of greatest height. The reason for this is that the conformal hodograph transform employed in the proof of the above theorem is no longer smooth, its only singularity being at the point corresponding to the wave crest, where the strict inequality in (2.8) becomes an equality. Since it is possible in this limiting case to give an exact description of the nature of this singularity, as one can see from the considerations made in [11, with some extra work one could obtain also the flow pattern beneath a solitary wave of greatest height. However, while in the case of smooth solitary waves there is a case to be made that the present approach is more elegant than the one pursued in [11, this claim does not extend to the case of a solitary wave of greatest height.

4. Comments. An area of current interest is the study of wave-current interaction, modelled by waves propagating on a flow with vorticity [10, 13, 15, 16, 17, 18, 24, 28, 41. While there is a variant of $\mathrm{KdV}$ appropriate for waves with vorticity 32 and rigorous results on small-amplitude solitary water waves with vorticity distribution were obtained recently [28, 30, the study of rotational solitary waves of large amplitude remains an open problem. Even for solitary waves of small amplitude, the presence of a non-zero vorticity precludes a strong reliance on harmonic function theory, in contrast to the present considerations made for irrotational flows. We expect a more intricate flow pattern beneath such solitary water waves. 


\section{REFERENCES}

[1] G. B. Airy, Tides and waves, Encyc. Metropolitana 5 (1845), 241-396.

[2] C. J. Amick, Bounds for water waves, Arch. Rat. Mech. Anal. 99 (1987), 91-114. MR886932 (88i:76009)

[3] C. J. Amick and J. F. Toland, On periodic water-waves and their convergence to solitary waves in the long-wave limit, Phil. Trans. Roy. Soc. London A 303 (1981), 633-669. MR647410 (83b:76009)

[4] C. J. Amick and J. F. Toland, On solitary water waves of finite amplitude, Arch. Rat. Mech. Anal. 76 (1981), 9-95. MR629699 (83b:76017)

[5] J. T. Beale, The existence of solitary water waves, Comm. Pure Appl. Math. 30 (1977), 373-389. MR 0445136(56:3480)

[6] M. J. Boussinesq, Théorie des ondes et des remous qui se propagent le long d'un canal réctangulaire horizontal, en communiquant au liquide contenu dans ce canal des vitesses sensiblement pareilles de la surface au fond, J. Math. Pures Appl. 17 (1872), 55-108.

[7] A. Constantin, On the deep water wave motion, J. Phys. A 34 (2001), 1405-1417. MR1819940 (2002b:76010)

[8] A. Constantin, Edge waves along a sloping beach, J. Phys. A 34 (2001), 9723-9731. MR1876166 (2002j:76015)

[9] A. Constantin, The trajectories of particles in Stokes waves, Inv. Math. 166 (2006), 523-535. MR 2257390 (2007j:35240)

[10] A. Constantin, M. Ehrnström and E. Wahlén, Symmetry of steady periodic gravity water waves with vorticity, Duke Math. J. 140 (2007), 591-603. MR.2362244

[11] A. Constantin and J. Escher, Particle trajectories in solitary water waves, Bull. Amer. Math. Soc. 44 (2007), 423-431. MR2318158 (2008m:76019)

[12] A. Constantin and R. S. Johnson, On the non-dimensionalisation, scaling and resulting interpretation of the classical governing equations for water waves, J. Nonl. Math. Phys. 15 (2008), 58-73. MR 2434725

[13] A. Constantin and R. S. Johnson, Propagation of very long water waves, with vorticity, over variable depth, with applications to tsunamis, Fluid Dynam. Res. 40 (2008), 175-211. MR.2369543

[14] A. Constantin and D. Lannes, The hydrodynamical relevance of the Camassa-Holm and DegasperisProcesi equations, Arch. Rat. Mech. Anal. 192 (2009), 165-186. MR2481064

[15] A. Constantin, D. Sattinger and W. Strauss, Variational formulations of steady water waves with vorticity, J. Fluid Mech. 548 (2006), 151-163. MR2264220 (2008b:76018)

[16] A. Constantin and W. Strauss, Exact steady periodic water waves with vorticity, Comm. Pure Appl. Math. 57 (2004), 481-527. MR2027299 (2004i:76018)

[17] A. Constantin and W. Strauss, Stability properties of steady water waves with vorticity, Comm. Pure Appl. Math. 60 (2007), 911-950. MR2306225

[18] A. Constantin and W. Strauss, Rotational steady water waves near stagnation, Phil. Trans. Roy. Soc. London A 365 (2007), 2195-2201. MR2329144 (2008j:76013)

[19] A. Constantin and W. Strauss, Pressure beneath a Stokes wave, Comm. Pure Appl. Math. DOI: 10.1002/cpa.20299.

[20] W. Craig, Non-existence of solitary water waves in three dimensions, Phil. Trans. Roy. Soc. London A 360 (2002), 2127-2135. MR1949966 (2003m:76011)

[21] W. Craig and P. Sternberg, Symmetry of solitary waves, Comm. Partial Differential Equations 13 (1988), 603-633. MR.919444 (88m:35132)

[22] A. D. D. Craik, The origins of water wave theory, Ann. Rev. Fluid Mech. 36 (2004), 1-28. MR 2062306 (2005a:01012)

[23] P. G. Drazin and R. S. Johnson, Solitons: an introduction, Cambridge University Press, Cambridge, 1989. MR985322 (90j:35166)

[24] M. Ehrnström, On the streamlines and particle paths of gravitational water waves, Nonlinearity 21 (2008), 1141-1154. MR2412330

[25] L. E. Fraenkel, An introduction to maximum principles and symmetry in elliptic problems, Cambridge University Press, Cambridge, 2000. MR,1751289 (2001c:35042)

[26] K. O. Friedrichs and D. H. Hyers, The existence of solitary waves, Comm. Pure Appl. Math. 7 (1954), 517-550. MR0065317 (16:413f)

[27] F. Gerstner, Theorie der Wellen samt einer daraus abgeleiteten Theorie der Deichprofile, Ann. Phys. 2 (1809), 412-445. 
[28] M. Groves and E. Wahlen, Small-amplitude Stokes and solitary gravity water waves with an arbitrary distribution of vorticity, Physica D 237 (2008), 1530-1538. MR2454604

[29] D. Henry, On Gerstner's water wave, J. Nonl. Math. Phys. 15 (2008), 87-95. MR2434727

[30] V. M. Hur, Exact solitary water waves with vorticity, Arch. Rat. Mech. Anal. 188 (2008), 213-244. MR.2385741

[31] R. S. Johnson, A modern introduction to the mathematical theory of water waves, Cambridge University Press, Cambridge, 1997. MR:1629555(99m:76017)

[32] R. S. Johnson, The classical problem of water waves: a reservoir of integrable and nearly-integrable equations J. Nonl. Math. Phys. 10 (2003), 72-92. MR2063546 (2005c:76018)

[33] D. J. Korteweg and G. deVries, On the change of form of long waves advancing in a rectangular canal and on a new type of long stationary waves, Phil. Mag. 39 (1895), 422-443.

[34] J. Lighthill, Waves in fluids, Cambridge University Press, Cambridge, 1978. MR642980 (84g:76001a)

[35] J. W. Miles, Solitary waves, Ann. Rev. Fluid Mech. 12 (1980), 11-43. MR.565388 (82e:76018)

[36] W. J. M. Rankine, On the exact form of waves near the surface of deep water, Phil. Trans. Roy. Soc. London A 153 (1863), 127-138.

[37] Lord Rayleigh, On waves, Phil. Mag. 1 (1876), 257-279.

[38] J. S. Russell, Report on waves, Rep. Meet. Brit. Assoc. Adv. Sci. 14 (1844), 311-390.

[39] J. J. Stoker, Water waves, Interscience Publ. Inc., New York, 1957. MR0103672 (21:2438)

[40] J. F. Toland, Stokes waves, Topol. Meth. Nonl. Anal. 7 (1996), 1-48. MR1422004 (97j:35130)

[41] E. Varvaruca, On some properties of traveling water waves with vorticity, SIAM J. Math. Anal. 39 (2008), 1686-1692. MR2377294 (2008m:76018) 